\title{
Attitude Control for a Micromechanical Flying Insect via Sensor Output Feedback
}

\author{
L. Schenato, W.C. Wu, and S.S. Sastry
}

\begin{abstract}
In this paper we study attitude stabilization strategies via output sensor feedback for Micro Aerial Vehicles (MAVs), inch-size robots capable of autonomous flight. To overcome the limited size and power budget available to these vehicles, ocelli and halteres, novel sensors based on body rotation and orientation sensing mechanisms used by flying insects, are introduced. The analysis and simulations of these sensors show the feasibility of using such biologically inspired approaches to build biomimetic gyroscopes and angular position detectors. Finally, attitude stabilization techniques based on these sensors are proposed and successfully tested on an aerodynamic model for a Micromechanical Flying Insect (MFI). To the authors' knowledge, this is the first attempt in using output feedback from biomimetic devices with ocelli and halteres to achieve attitude stabilization in MAVs.
\end{abstract}

\section{INTRODUCTION}

$\mathbf{M}$ ICRO aerial vehicles (MAVs) have drawn a great deal of attention in the past decade due to the rapid advances in microtechnology. Several groups have worked on MAVs with fixed and rotary wings [1]. Flapping flight, however, provides superior maneuverability that is beneficial in obstacle avoidance and navigation in small spaces. Therefore, the UC Berkeley Micromechanical Flying Insect (MFI) project uses biomimetic principles to develop a flapping wing MAV that will be capable of sustained autonomous flight [2],[3]. One important concern in designing the components of the MFI is their power consumption and size. Current power budget for the MFI is $25 \mathrm{~mW}$, and the majority of this power will be allocated to the actuation of the two wings.

On the other hand, the sensory system of the MFI, which is crucial for stabilizing flight, should consume little power. The power requirements of off-the-shelf micro sensors are generally too expensive for the MFI. At present, piezo-actuated biomimetic angular rate sensors for use on the MFI have been constructed [4]. Another biomimetic device, ocelli, has been fabricated [5]. Ocelli consist of four photoreceptors that sense the light intensity of surrounding areas in order to estimate the orientation of the MFI. Both sensors have the advantages of simple design, easy implementation, low power consumption, and high performance. This paper first presents the modeling of these two biologically inspired sensors and then proposes a close-loop attitude control scheme using the sensor output as feedback.

\section{FLying INSECT DYNAMICS}

Flight dynamics of flapping insects is still an open area of research [6]. This is primarily due to the difficulties in measuring

L. Schenato, W.C. Wu, and S. Sastry are with the Department of Electrical Engineering and Computer Sciences, University of California, Berkeley, CA 94720, USA (email:\{lusche,wcwu,sastry\}@eecs.berkeley.edu) aerodynamic forces on real flying insects, and in experimentally validating proposed theoretical models. In this work we model the dynamics of a flying insect as a rigid body subject to external forces. Albeit wings do move relative to the insect body, their mass is within $1-5 \%$ of total insect mass and hence their effect on the insect dynamics is relatively small and can be neglected. Therefore, we assume that the insect body motion evolves according to the rigid body motion equations subject to external forces relative to its center of mass [7]. The external forces acting on an insect are the aerodynamic forces generated by the wings, the gravity force, and the body viscous drag. Since we are interested in attitude control, gravity does not play a role. Also, since only slow body rotations are considered in this work, angular viscous forces are neglected. Finally, we assume that the aerodynamic torques can be controlled exactly and continuously. In reality, the aerodynamic forces generated by flapping wings are highly time-varying within a single wingbeat and they can not be controlled instantaneously. Current research is aimed at solving this problem and promising preliminary results have been shown [8] [9]. These results will soon be applied to the control schemes proposed in this paper.

Summing up, the dynamics of the attitude of a flapping insect are modeled as follows:

$$
\begin{aligned}
\dot{R} & =R \hat{\omega}^{b} \\
\dot{\omega}^{b} & =J_{b}^{-1}\left(\boldsymbol{\tau}^{b}-\boldsymbol{\omega}_{b} \times J_{b} \boldsymbol{\omega}_{b}\right) \\
\boldsymbol{\tau}^{b} & =u \\
\hat{\omega}^{b} & =\left[\begin{array}{ccc}
0 & \omega_{z}^{b} & -\omega_{y}^{b} \\
-\omega_{z}^{b} & 0 & \omega_{x}^{b} \\
\omega_{y}^{b} & -\omega_{x}^{b} & 0
\end{array}\right]
\end{aligned}
$$

where $\boldsymbol{\omega}^{b}=\left[\omega_{x}^{b} \omega_{y}^{b} \omega_{z}^{b}\right]^{T}$ is the angular velocity of the insect body relative to the body frame $B, \tau^{b} \in \mathbb{R}^{3}$ is the total external torque relative to the body frame $B$ attached to the center of mass of the insect body, $J_{b} \in \mathbb{R}^{3 \times 3}$ is the moment of inertia of the insect body relative to the body frame $B$, and $\mathbf{u} \in \mathbb{R}^{3}$ is the control input vector. To simplify the notation, we drop the superscript $b$ from equations, implicitly assuming that all quantities are measured relative to the body frame $B$. The matrix $R \in S O(3)=\left\{R \in \mathbb{R}^{3 \times 3}: R^{T} R=I\right.$, det $\left.R=+1\right\}$ is the rotation matrix representing the orientation of the insect body frame $B$ relative to the fixed frame $A$. In particular, let $\mathbf{v}^{b}=\left[x_{b} y_{b} z_{b}\right]^{T}$ and $\mathbf{v}^{a}=\left[x_{a} y_{a} z_{a}\right]^{T}$ the coordinates of a vector $\mathbf{v} \in \mathbb{R}^{3}$ relative to the body frame $B$ and the fixed frame $A$, respectively. Then, these coordinates satisfy the following transformations:

$$
\begin{aligned}
& \mathbf{v}_{a}=R \mathbf{v}_{b} \\
& \mathbf{v}_{b}=R^{T} \mathbf{v}_{a}
\end{aligned}
$$




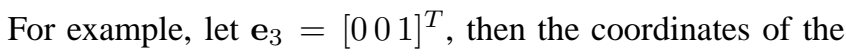
$\mathrm{z}$-axis unit vector of the body frame relative to the fixed frame are $P_{z}^{a}=R \mathbf{e}_{3}=\left[r_{13} r_{23} r_{33}\right]^{T}$, while the the coordinates of the $\mathrm{z}$-axis unit vector of the fixed frame relative to the body frame are $P_{z}^{b}=R^{T} \mathbf{e}_{3}=\left[\begin{array}{ll}r_{31} & r_{32} r_{33}\end{array}\right]^{T}$. In general, $P_{z}^{b} \neq P_{z}^{a}$. Moreover, from the definition of rotation matrix and Equation (1a), we have the following useful properties:

$$
\begin{aligned}
\left\|P_{z}^{b}\right\|^{2} & =r_{31}^{2}+r_{32}^{2}+r_{33}^{2}=1 \\
\dot{P}_{z}^{b} & =P_{z}^{b} \times \boldsymbol{\omega}
\end{aligned}
$$

This model is very similar to satellite dynamics [10] and control strategies can be applied [11] [12] [13] [14].

\section{OCELLI}

\section{A. Morphology and Purpose}

Ocelli are a sensory system present in many flying insects. This system comprises of three wide angle photoreceptors placed on the head of the insect (see Figure 1). They are oriented in such a way that they collect light from different regions of the sky (see Figure 2), but have poor image resolution. Although the exact physiology and purpose of ocelli and their purpose in insect flight are still not completely understood, it is believed that they play a fundamental role in insect attitude stabilization, and particularly horizon stabilization [15] [16] [17] [18]. Experimental results performed by Taylor [17] and Kastberger [16] on some insect species suggest that ocelli collaborate synergistically with compound eyes to minimize the delay of visual processing and to augment visual responsiveness when no sharp horizontal border is present. When an insect is presented with a moving artificial horizon, it first tries to rotate its head in order to fixate the horizon on the retina. Only afterwards does it change its wing pattern to realign its abdomen with its head. Taylor observed that cauterization of ocelli doubles the latency between the horizon motion, and the compensatory head movement. Moreover, in dimly lit environments ablated ocelli also reduced insect sensitivity to horizon motions resulting in smaller mean amplitude of head motion responses. Therefore it can be stated that ocelli are especially important for stabilization of the retinal image of the compound eyes during flight, when disturbances are sudden and frequent. Ocelli seem to be designed for high sensitivity and speed of response at the expense of acuity. Two additional findings deserve mentioning. The first finding is that, in case of ablated compound eyes and intact ocelli, an inverted horizon, corresponding to upside-down insect orientation, caused no head motion unless the two lateral ocelli were unequally illuminated, unlike insects with intact compound eyes. This is consistent with the mathematical modeling of ocelli developed in the following paragraphs, which predicts an unstable equilibrium configuration for the upside-down orientation. The second finding is that the insect head with compound eyes disconnected and intact ocelli, quickly responded to sudden horizon displacements, but then soon relaxed toward the rest position even when the horizon remained displaced. In other cases, animals with intact compound eyes maintained a rotated head. This observation suggests that the ocelli behave similarly to a high pass filter. This could be motivated by the fact that light distribution can change substantially during the course of the day due to sun motion, atmospheric variations or simply because the insect during its flight can traverse shady trees or urban environments. Nonetheless, these variations have a long timescale relative to the insect motion timescale and can be compensated by the compound eyes. From an engineering perspective, insects combine lowbandwidth compound eyes with high-bandwidth ocelli to obtain an accurate horizon sensor for attitude stabilization over a large frequency domain.

Biologists believe that ocelli estimate the orientation of the insect with respect to the sky by comparing the intensity of light measured by the different photoreceptors. Their argument is based on the assumption that, as a first approximation, the intensity of light measured by the photoreceptors, $I$, is only a function of its latitude $\theta$ relative to the light source (i.e. the sun).

In this paper, we consider ocelli systems with four photoreceptors rather than three, as for real insects. Although all the results in this paper can be extended to a three-photoreceptor ocelli, we prefer to present them relative to a four-photoreceptor configuration since proofs are more elegant and intuitive.

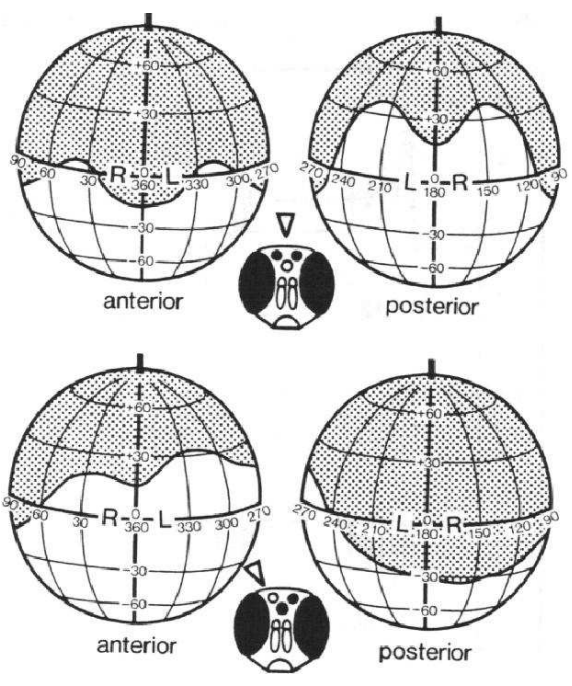

Fig. 1. The ocelli of a blowfly and the visual fields of the median (top) and right lateral (bottom) ocelli. Courtesy of [15].

Before entering the discussion of ocelli modeling, it is important to mention that some horizon sensors for attitude stabilization are commercially available. The most interesting ones, such as the FMA co-pilot, and the Futaba PA-2, for Radio Controlled aircrafts, are based on 4 optical sensors on two-axis planar configuration that sense the difference in infrared signature between the earth and the carbon dioxide in the atmosphere to provide day and night roll-pitch stabilization. Despite their exact functioning is not available to the public, they seem very much ocelli-like in function, since they rely on the differential measurements of a monotonic function of the vertical latitude. Therefore, we believe they may fall within the ocelli mathematical modeling developed in this paper. The major difference between a carbon-dioxide-based ocelli and a light-intensity-based ocelli is that the former one cannot work in indoor environments 

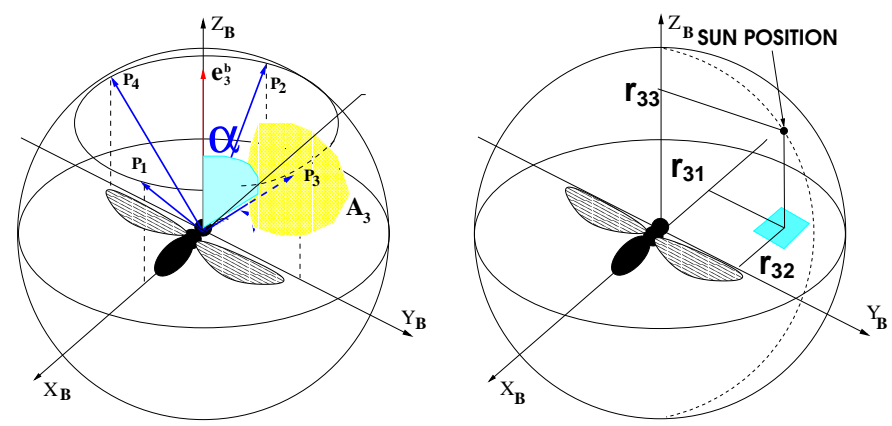

Fig. 2. (a) Four photoreceptors, $P_{1}, P_{2}, P_{3}$, and $P_{4}$, are fixed with respect to the insect's body frame $\left(x_{B}, y_{B}, z_{B}\right)$. The shadowed area, $A_{3}$, represents the receptive region of $P_{3}$; (b) The projection of the light source onto the $x-y$ plane of the insect's body frame. The shadowed area represents the box given by the inequalities in Equation (15).

and it is likely to fail in urban or shady environments when part of the sky is occluded.

\section{B. Modeling}

Any point $P$ in the sky can be represented in spherical coordinates $(r, \theta, \psi)$ where $r \in[0,+\infty]$ is the radius of the celestial sphere, $\theta \in[0, \pi]$ is the latitude, and $\psi \in[0,2 \pi]$ is the longitude, relative to the fixed frame $A$. Alternatively, the same point can be written in Cartesian coordinates $P=\left[\begin{array}{ll}x_{P} & y_{P} \\ z_{P}\end{array}\right]^{T}$. The transformation from spherical to Cartesian coordinates is given by:

$$
\begin{aligned}
& x_{P}=r \sin \theta \cos \psi \\
& y_{P}=r \sin \theta \sin \psi \\
& z_{P}=r \cos \theta
\end{aligned}
$$

Without loss of generality, the radius of the celestial sphere can be normalized to unity, i.e. $r=1$. The ocelli sensory system is modeled as four ideal photoreceptors, called $P_{1}, P_{2}, P_{3}, P_{4}$, fixed with respect to the body frame $B$, that collect the light intensity from a region of the sky. They are oriented symmetrically such that they have the same latitude and their axes intersect the sky sphere forming an imaginary pyramid, whose vertex is placed at the center of the insect head. Formally, their orientation relative to the body frame $B$ can be represented in Cartesian coordinates as follows:

$$
\begin{array}{ll}
P_{1}^{b}=\left[\sqrt{1-h^{2}} 0 h\right]^{T}, & P_{2}^{b}=\left[-\sqrt{1-h^{2}} 0 h\right]^{T} \\
P_{3}^{b}=\left[0 \sqrt{1-h^{2}} h\right]^{T}, & P_{4}^{b}=\left[0-\sqrt{1-h^{2}} h\right]^{T}
\end{array}
$$

where the parameter $h \in(-1,1)$ sets the latitude of the photoreceptors. Every photoreceptor collects light from a conic region $A_{i}$ around its ideal orientation $P_{i}$ as shown in Figure 2.

The most important assumption made in ocelli modeling is that the intensity of light, $I$, measured by a photoreceptor $P$, is independent of its longitude and it is a strictly monotonically decreasing function of only its latitude relative to the fixed frame. Formally it can be written as:

$$
\begin{gathered}
I(P) \quad=\quad I(\psi, \theta)=I(\theta) \\
\theta_{1}<\theta_{2} \Rightarrow I\left(\theta_{1}\right)>I\left(\theta_{2}\right)
\end{gathered}
$$
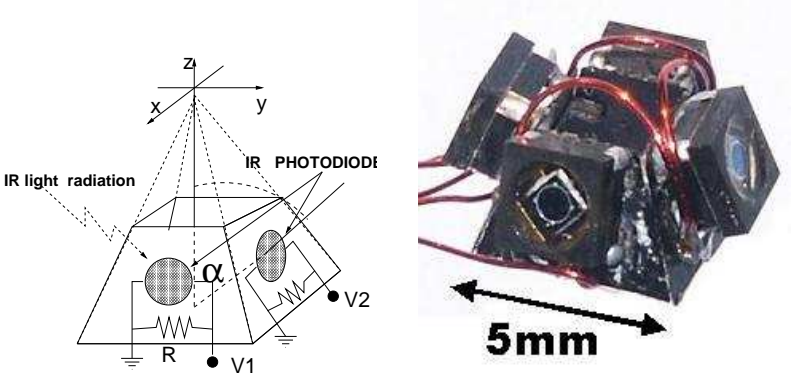

Fig. 3. (a) Schematics of ocelli design; (b) Photo of ocelli prototype. Adapted from [5].

where, with an abuse of notation, we identify the position of the photoreceptor with its latitude $\theta$, which is the angle between the $\mathrm{z}$-axis of the fixed frame $A$ and the orientation of the photoreceptor on the celestial sphere.

The monotonic distribution of light intensity on the celestial sphere can be satisfied only in an ideal environment where the landscape is uniform and the light is diffused uniformly around its generating source. In reality, time-varying atmospheric conditions, trees and building creating shady regions, or multiple light sources in indoor environments can undermine this assumption. In order to test this assumption, we built a small prototype of ocelli using off-the-shelf photodiodes with peak sensitivity at light wavelength $900 \mathrm{~nm}$, conic field of view of $60^{\circ}$ diameter and $0.75 \mathrm{~mm}^{2}$ active area. These photodiodes are placed on a square pyramid with angle $\alpha=40^{\circ}$ (see Figure 3). Design details can be found in [5]. The ocelli prototype was then placed about $1 \mathrm{~m}$ from the ground in three different environments that represent typical range of MFI scenarios: inside a room illuminated by multiple lamps on the ceiling, outdoor between buildings that were blocking the sun, and outdoor in a open space during a cloudy day. The output from a single photodiode placed at different orientation was used to generate the light intensity map of the celestial sphere shown in Figure 4. To facilitate the comparison of light intensity for heterogeneous environments, the intensity in these three plots was normalized so that $I_{\max }=1, I_{\min }=-1$. Although the light intensity is not strictly monotonic and it depends also on the longitude, it is still possible to spot a bright portion of the sky opposed to a dark one in all three scenarios. In the indoor environment, the bright region is exactly perpendicular to horizontal plane, while in the outdoor scenarios it is slightly tilted. In particular, in the urban environment case, the bright area is tilted because a large building is screening the sun, while in the open area environment the tilt is caused by the position of the sun close to the horizon (see photos on the left side in Figure 4). The consequences on the attitude estimation of ocelli caused by these non ideal scenarios will be addressed later.

The measurements from the photoreceptors are simply subtracted pairwise and these two signals are the output from the ocelli:

$$
\begin{aligned}
& y_{1}=I\left(P_{1}^{a}\right)-I\left(P_{2}^{a}\right) \\
& y_{2}=I\left(P_{3}^{a}\right)-I\left(P_{4}^{a}\right)
\end{aligned}
$$

where $P_{i}^{a}$ is the photoreceptor orientation in Cartesian coordinates relative to the fixed frame $A$. Given the orientation 

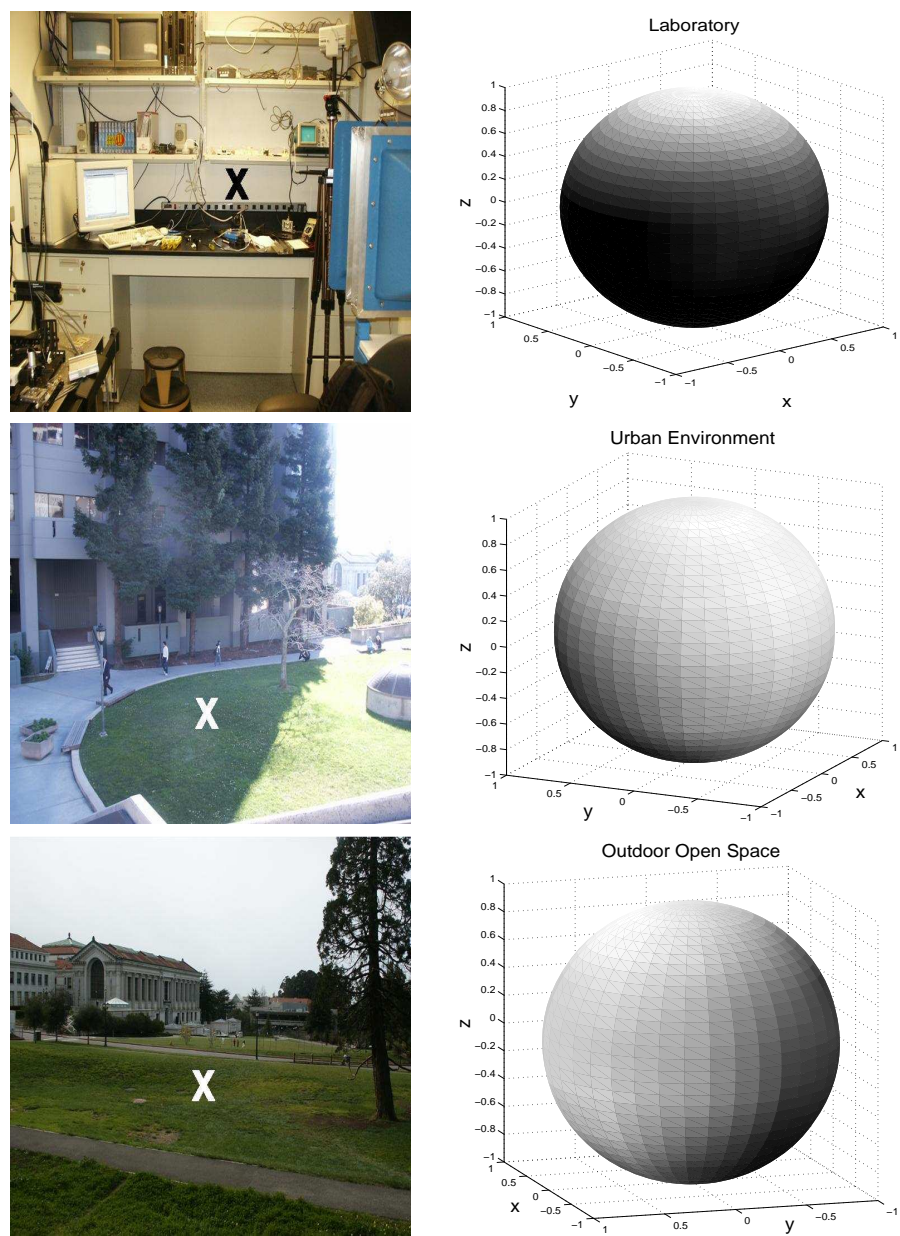

Urban Environment
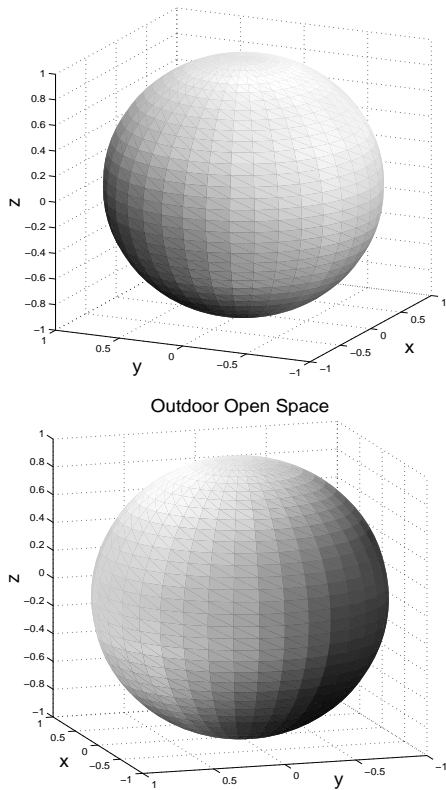

Fig. 4. Light intensity distribution over the celestial sphere from experimental data: indoor environment (top), urban environment (center), outdoor open space environment(bottom). Experimental plots are obtained by interpolating (but not smoothing) the light intensity measured by the ocelli on a uniform grid of 100 points collected on locations marked by the cross "X" on the pictures.

$R \in S O(3)$, of the insect body frame $B$ relative to the fixed frame $A$, the orientation of the photoreceptor $P_{i}$ relative to the fixed frame is $P_{i}^{a}=R P_{i}^{b}$. Since the orientation of the photoreceptors is fixed with respect to the body frame $B$, the outputs from the ocelli depend only on the insect orientation $R$. From a mathematical point of view, the ocelli system can be modeled as a nonlinear function $f: S O(3) \rightarrow \mathbb{R}^{2}$ of the insect orientation.

\section{Orientation Estimation}

This section is devoted to studying the general properties of the map $f()$, i.e. how much information about the orientation $R$ can be extracted from the ocelli output. We first consider the special case where the light intensity measured by the photoreceptors is $I(\theta)=\cos \theta$. This instance clearly highlights the relation between the insect orientation $R$ and the ocelli output $y$. Then we consider the general case where $I(\theta)$ is simply monotonic.

Proposition 1. Suppose that the light intensity measured by the photoreceptors is $I(\theta)=\cos \theta$, and let the orientation of the photoreceptors be such that $h=\frac{\sqrt{3}}{2}$ in Equations (6). Then the output of the ocelli is $y_{1}=r_{31}, y_{2}=r_{32}$, where $r_{i j}$ is the $i-j$ entry of the insect orientation matrix $R$.

Proof: Substituting $h=\frac{\sqrt{3}}{2}$ and $I(\theta)=\cos \theta$ into Equations (8) we get:

$$
\begin{aligned}
y_{1} & =I\left(P_{1}^{a}\right)-I\left(P_{2}^{a}\right)=\cos \theta_{P_{1}^{a}}-\cos \theta_{P_{2}^{a}} \\
& =\mathbf{e}_{3}^{T} P_{1}^{a}-\mathbf{e}_{3}^{T} P_{2}^{a}=\mathbf{e}_{3}^{T} R P_{1}^{b}-\mathbf{e}_{3}^{T} R P_{2}^{b} \\
& =\mathbf{e}_{3}^{T} R\left(P_{1}^{b}-P_{2}^{b}\right)=\mathbf{e}_{3}^{T} R \mathbf{e}_{1}=r_{31} \\
y_{2} & =\ldots=\mathbf{e}_{3}^{T} R\left(P_{3}^{b}-P_{4}^{b}\right)=\mathbf{e}_{3}^{T} R \mathbf{e}_{2}=r_{32}
\end{aligned}
$$

where $\mathbf{e}_{1}=\left[\begin{array}{lll}1 & 0 & 0\end{array}\right]^{T}, \mathbf{e}_{2}=\left[\begin{array}{lll}0 & 1 & 0\end{array}\right]^{T}, \mathbf{e}_{3}=\left[\begin{array}{lll}0 & 0 & 1\end{array}\right]^{T}$. The second line follows from the fact that $\cos \theta_{P^{a}}=z_{P}=\mathbf{e}_{3}^{T} P^{a}$ and that $P_{i}^{a}=R P_{i}^{b}$.

As described at the end of Section II, $r_{31}$ and $r_{32}$ correspond to the $x$ and $y$ coordinates of the $z$-axis of the fixed frame $A$ relative to the body frame $B$. In other words, the ocelli can measure the $x$ and $y$ position of the light source relative to the insect body. Intuitively, it is clear that this information can be used to rotate the insect body toward the light source.

When the light intensity $I(\theta)$ measured by the photoreceptors is just a monotonically decreasing function of the latitude, the ocelli do not estimate the exact orientation of the sun relative to the insect body frame, but they can still retrieve the approximate direction, as shown in the following proposition:

Proposition 2. Suppose that the light intensity measured by the photoreceptors, $I(\theta)$, is an unknown strictly monotonically decreasing function of the latitude $\theta$. Then the output of the ocelli has the following properties:

$$
\begin{aligned}
& y_{1}=0 \Longleftrightarrow r_{31}=0 ; \quad y_{1} \neq 0 \Longrightarrow y_{1} r_{31}>0 \\
& y_{2}=0 \Longleftrightarrow r_{32}=0 ; \quad y_{2} \neq 0 \Longrightarrow y_{2} r_{32}>0
\end{aligned}
$$

Proof: First we recall that $\cos ^{-1}()$ is a strictly monotonically decreasing function of its argument, and that the composition of two monotonically decreasing functions is a monotonically increasing function. Thus, $\tilde{I}(\theta)=I \circ \cos ^{-1}(\theta)$ is a monotonically increasing function. Consider the first ocelli output $y_{1}$ :

$$
\begin{aligned}
y_{1} & =I\left(\theta_{P_{1}^{a}}\right)-I\left(\theta_{P_{2}^{a}}\right) \\
& =I\left(\cos ^{-1}\left(\mathbf{e}_{3}^{T} P_{1}^{a}\right)\right)-I\left(\cos ^{-1}\left(\mathbf{e}_{3}^{T} P_{2}^{a}\right)\right) \\
& =\tilde{I}\left(\mathbf{e}_{3}^{T} P_{1}^{a}\right)-\tilde{I}\left(\mathbf{e}_{3}^{T} P_{2}^{a}\right) \\
& =\tilde{I}\left(\mathbf{e}_{3}^{T} R P_{1}^{b}\right)-\tilde{I}\left(\mathbf{e}_{3}^{T} R P_{2}^{b}\right) \\
& =\tilde{I}\left(r_{31} \sqrt{1-h^{2}}+r_{33} h\right)-\tilde{I}\left(-r_{31} \sqrt{1-h^{2}}+r_{33} h\right)(10)
\end{aligned}
$$

where we use the fact $\cos \theta_{P^{a}}=z_{P}=\mathbf{e}_{3}^{T} P^{a}$ in the second line, and the orientations $P_{i}^{b}$ of the photoreceptors are given by Equations (6). Let us define $l=\sqrt{1-h^{2}}$. Since the function $\tilde{I}$ is monotonically increasing we have:

$$
\begin{aligned}
y_{1}>0 & \Rightarrow \tilde{I}\left(r_{31} l+r_{33} h\right)>\tilde{I}\left(-r_{31} l+r_{33} h\right) \\
& \Rightarrow r_{31} l+r_{33} h>-r_{31} l+r_{33} h \\
& \Rightarrow 2 r_{31} l>0 \Rightarrow r_{31}>0
\end{aligned}
$$

where we use the fact that $\tilde{I}$ is monotonically increasing and that $l>0$. Analogously, it is easy to verify that $y_{1}<0 \Rightarrow$ $r_{31}<0$. From monotonicity of $\tilde{I}$ also follows that $y_{1}=0 \Rightarrow$ $r_{31}=0$. Trivially, from Equation (10) it follows that $r_{31}=$ 
$0 \Rightarrow y_{1}=0$. Finally, the same arguments can be used to prove the properties of ocelli output $y_{2}$.

This proposition indicates that the ocelli still give an approximate orientation of the light source, regardless of the exact orientation of the photoreceptors relative to the insect body and regardless of the specific light intensity distribution as long as it is monotonic. Moreover, the outputs of the ocelli are zero if and only if the $z$-axis of the fixed and body frame are aligned. A more intuitive understanding of the ocelli processing is given by the following lemma:

Lemma 1. Suppose that the light intensity measured by the photoreceptors, $I(\theta)$, is an unknown smooth differentiable monotonically decreasing function of the latitude $\theta$. Let $P_{z}^{b}=$ $\left[\begin{array}{lll}r_{31} & r_{32} & r_{33}\end{array}\right]^{T}$ represent the orientation of the z-axis of the fixed frame relative to the body frame, and let $\psi_{b}$ and $\theta_{b}$ represent the longitude and latitude of the vector $P_{z}^{b}$ relative to the body frame, respectively. Also let $\hat{\psi}_{b}$ represent the longitude of the vector $\mathbf{y}_{O}=\left[\begin{array}{lll}y_{1} & y_{2} & 0\end{array}\right]^{T}$. Then we have:

$$
\begin{aligned}
& \left|\hat{\psi}_{b}-\psi_{b}\right|<\frac{\pi}{2}, \text { for } \mathbf{y}_{O} \neq 0 \\
& \underline{a} r_{31} \leq y_{1} \leq \bar{a} r_{31} ; \quad \underline{a} r_{32} \leq y_{2} \leq \bar{a} r_{32} \\
& \theta_{b} \rightarrow 0 \Longrightarrow\left\{\begin{array}{rll}
y_{1} & \rightarrow a r_{31} \\
y_{2} & \rightarrow a r_{32} \\
\hat{\psi}_{b} & \rightarrow & \psi_{b} \\
\left\|\mathbf{y}_{O}\right\| & \rightarrow & a \sin \theta_{b}
\end{array}\right.
\end{aligned}
$$

where $0<\underline{a} \leq a \leq \bar{a}<\infty$

Proof: According to the definition of longitude given in Equation (5), we have $\sin \psi_{b}=\frac{r_{31}}{\sqrt{r_{31}^{2}+r_{32}^{2}}}, \cos \psi_{b}=$ $\frac{r_{32}}{\sqrt{r_{31}^{2}+r_{32}^{2}}}, \sin \hat{\psi}_{b}=\frac{y_{1}}{\sqrt{y_{1}^{2}+y_{2}^{2}}}, \cos \hat{\psi}_{b}=\frac{y_{2}}{\sqrt{y_{1}^{2}+y_{2}^{2}}}$. Therefore, if $\left(y_{1}, y_{2}\right) \neq(0,0)$, we have:

$$
\begin{aligned}
\cos \left(\psi_{b}-\hat{\psi}_{b}\right) & =\cos \psi_{b} \cos \hat{\psi}_{b}+\sin \psi_{b} \sin \hat{\psi}_{b} \\
& =\frac{r_{31} y_{1}+r_{32} y_{2}}{\sqrt{\left(y_{1}^{2}+y_{2}^{2}\right)\left(r_{31}^{2}+r_{32}^{2}\right)}}>0
\end{aligned}
$$

where the inequality follows from Proposition 2, and it implies Equation (11). For $\mathbf{y}_{O}=0$, the longitude is ill-defined since it corresponds to a point of singularity of the spherical coordinate representation.

Following from Equation (10), if $r_{31}>0$ we have:

$$
\begin{aligned}
y_{1}= & \tilde{I}\left(h r_{33}\right)+r_{31} \sqrt{1-h^{2}} \frac{d \tilde{I}\left(\xi_{1}\right)}{d \theta}-\left[\tilde{I}\left(h r_{33}\right)-\right. \\
& \left.-r_{31} \sqrt{1-h^{2}} \frac{d \tilde{I}\left(\xi_{2}\right)}{d \theta}\right]=\sqrt{1-h^{2}}\left[\frac{d \tilde{I}\left(\xi_{1}\right)}{d \theta}+\frac{d \tilde{I}\left(\xi_{2}\right)}{d \theta}\right] r_{31} \\
\Longrightarrow & 2 \sqrt{1-h^{2}} \underline{a} r_{31} \leq y_{1} \leq 2 \sqrt{1-h^{2}} \bar{a} r_{31}
\end{aligned}
$$

where $\xi_{1} \in\left[h r_{33}, h r_{33}+r_{31} \sqrt{1-h^{2}}\right], \xi_{2} \in\left[h r_{33}-\right.$ $\left.h_{31} \sqrt{1-h^{2}}, h r_{33}\right]$ come from the mean value function theorem, and the inequalities from the assumption that $0<\underline{b} \leq$ $\frac{d \tilde{I}}{d \theta} \leq \bar{b}<\infty$ is smooth with nonnegative bounded first derivative. These inequalities lead directly to Equations (12).

According to Equations (5), we have $r_{33}=\cos \theta_{b},\left|r_{31}\right| \leq$ $\sin \theta_{b},\left|r_{32}\right| \leq \sin \theta_{b}$, therefore $\left(\theta_{b} \rightarrow 0\right) \Rightarrow\left(P_{z}^{b}=\right.$ $\left.\left[r_{31} r_{32} r_{33}\right] \rightarrow \mathbf{e}_{3}=\left[\begin{array}{lll}0 & 0 & 1\end{array}\right]\right)$. Also $\left(\theta_{b} \rightarrow 0\right) \Rightarrow\left(\xi_{1} \rightarrow\right.$
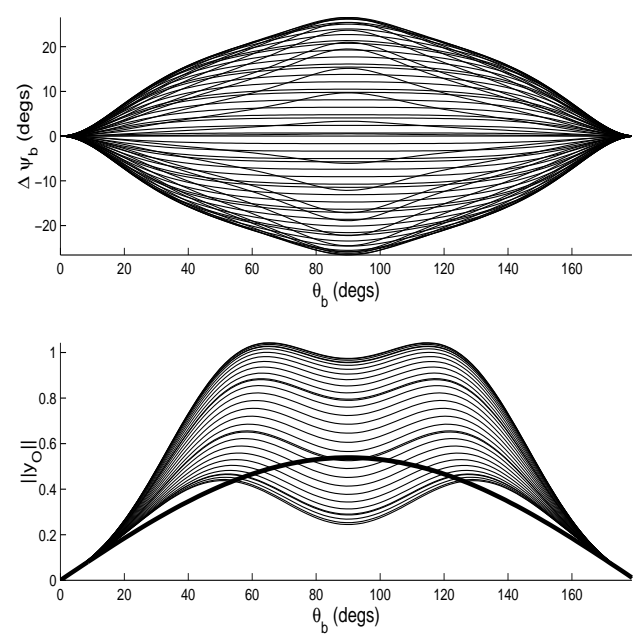

Fig. 5. Longitude estimation error, $\Delta \psi_{b}=\psi_{b}-\hat{\psi}_{b}$, and magnitude of ocelli output, $\left\|\mathbf{y}_{O}\right\|$, as a function of latitude $\theta$ of the light source position relative to the insect body for light intensity $I=\cos ^{5}(\theta)$. Each trace corresponds to a different longitude $\psi$. The thick line in lower plot is given by function $f=a \sin \theta_{b}$ of Equation (13)

$\left.h ; \xi_{1} \rightarrow h\right)$, therefore, because of Equation (15), $\left(y_{1} \rightarrow a r_{31}\right)$ where $a=2 \frac{d \tilde{I}(h)}{d \theta} \sqrt{1-h^{2}}$. Analogously, $\left(\theta_{b} \rightarrow 0\right) \Rightarrow\left(y_{2} \rightarrow\right.$ $\left.\operatorname{ar}_{32}\right)$.

Equation (14) can be expanded in Taylor's series relative to the variables $P_{z}^{b}=\left[r_{31} r_{32} r_{33}\right]$ at the point $\mathbf{e}_{3}$ :

$$
\begin{aligned}
\cos \left(\psi_{b}-\hat{\psi}_{b}\right) & =\frac{a r_{31}^{2}+a r_{32}^{2}}{\sqrt{a^{2}\left(r_{31}^{2}+r_{32}^{2}\right)\left(r_{31}^{2}+r_{32}^{2}\right)}}+o\left(\left\|P_{z}^{b}-\mathbf{e}_{3}\right\|\right) \\
& =1+o\left(\left\|P_{z}^{b}-\mathbf{e}_{3}\right\|\right)
\end{aligned}
$$

therefore $\theta_{b} \rightarrow 0 \Rightarrow\left\|P_{z}^{b}-\mathbf{e}_{3}\right\| \rightarrow 0 \Rightarrow \cos (\psi-\hat{\psi}) \rightarrow 1 \Rightarrow$ $(\psi-\hat{\psi}) \rightarrow 0$. Finally, the magnitude of the output vector can be written as

$$
\begin{aligned}
\left\|\mathbf{y}_{O}\right\| & =\sqrt{y_{1}^{2}+y_{2}^{2}}=a \sqrt{r_{31}^{2}+r_{32}^{2}}+o\left(\left\|P_{z}^{b}-\mathbf{e}_{3}\right\|\right) \\
& =a \sqrt{1-r_{33}^{2}}+o\left(\left\|P_{z}^{b}-\mathbf{e}_{3}\right\|\right) \\
& =a \sqrt{1-\cos ^{2} \theta_{b}}+o\left(\left\|P_{z}^{b}-\mathbf{e}_{3}\right\|\right) \\
& =a \sin \theta_{b}+o\left(\left\|P_{z}^{b}-\mathbf{e}_{3}\right\|\right)
\end{aligned}
$$

where we used the identity $r_{31}^{2}+r_{32}^{2}+r_{33}^{2}=1$. Therefore, $\theta_{b} \rightarrow 0 \Rightarrow\left\|P_{z}^{b}-\mathbf{e}_{3}\right\| \rightarrow 0 \Rightarrow\left\|\mathbf{y}_{O}\right\| \rightarrow a \sin \theta_{b}$.

This lemma highlights several important features of ocelli outputs. First, according to Equation (11), the ocelli always give an approximate estimation of the latitude of the light source relative to the insect body, in the sense that if the insect rotates toward the apparent position of the light source given by the ocelli, it will eventually align the $z$-axes of the body and fixed frame. Also, for small latitudes $\theta_{b}$, the longitude error of the light source decreases to zero, and the magnitude of the output vector $\mathbf{y}_{O}$ becomes proportional to the latitude. This means that the ocelli outputs not only estimate the direction of the light source but also its distance in terms of the latitude.

Figure 5 gives a pictorial representation of ocelli outputs for the light intensity function $I(\theta)=\cos ^{5} \theta$. The plot on the top 

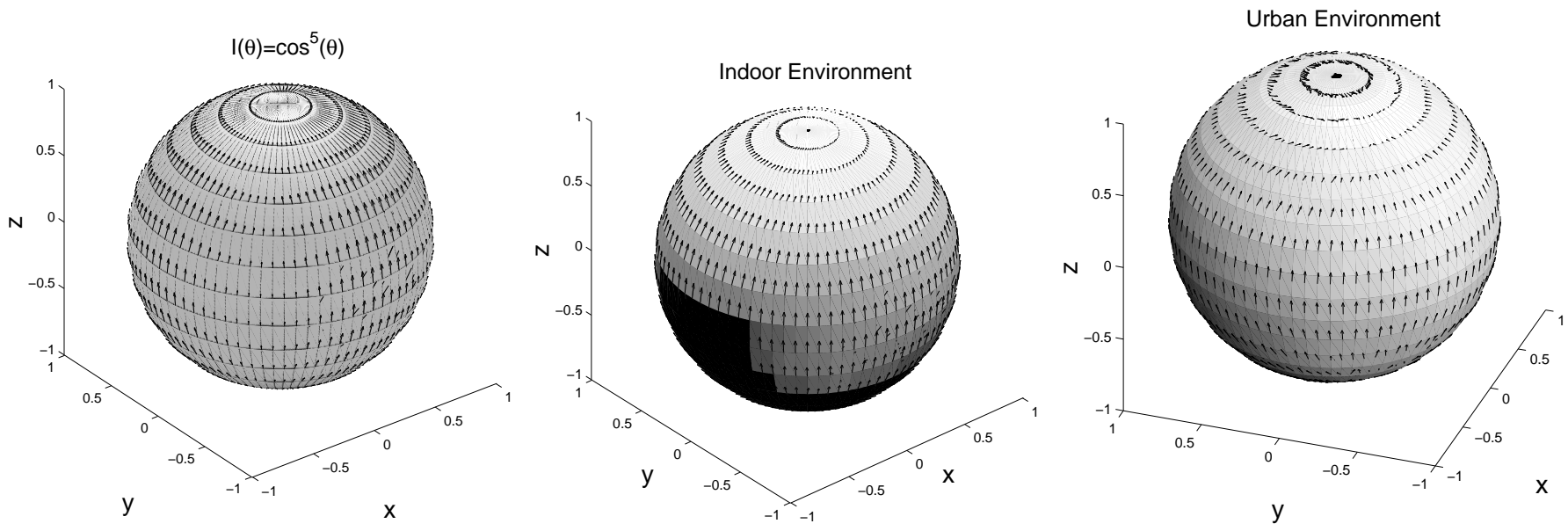

Fig. 6. Force field relative to the body frame induced by the ocelli for different light intensity distributions: ideal monotonic distribution $I(\theta)=\cos ^{5} \theta($ left $)$; measured indoor environment (center); measured urban environment (right). The light intensity distributions of center and right plots are the same as the top and the center plots shown Figure 4.

displays the latitude error $\psi$ against the latitude for different longitudes. The plot on the bottom displays the magnitude of the output vector as a function of the latitude for different longitudes. As expected, the longitude error is always smaller than $90^{\circ}$ and goes to zero as the latitude goes to zero. The magnitude of the output vector is always positive except for the two points $\theta_{b}=\{0, \pi\}$, and it is clearly proportional to the sine of the latitude for small angles.

An additional graphical representation of the ocelli performance as an estimator of the light source position is given by the virtual torque field induced by the ocelli output as if the body frame were attracted toward the apparent light source position with magnitude proportional to the ocelli output. More formally, this induced torque field can be written as:

$$
\boldsymbol{\tau}_{y}=-\mathbf{e}_{3} \times \mathbf{y}_{O}=\left[\begin{array}{c}
-y_{2} \\
y_{1} \\
0
\end{array}\right] ;
$$

where $\mathbf{e}_{3}=\left[\begin{array}{lll}0 & 0 & 1\end{array}\right]^{T}$. The left plot in Figure 6 shows the virtual force field relative to the body frame, which is given by $\mathbf{F}_{y}=P_{z}^{b} \times \boldsymbol{\tau}_{y}$, for the light intensity function $I(\theta)=\cos ^{5} \theta$. Clearly, this field would eventually move the insect body frame toward the north pole, although the magnitude and the direction change for different latitudes and longitudes. We also estimated the virtual force field relative to the light intensity measured in the three scenarios of Figure 4. The force field for the indoor setting was very close to the ideal case where all vectors were pointing directly to the north pole (see center plot of Figure 6). Also in the outdoor settings the vector field points to the position of the apparent light source, as it can be seen in the right plot of Figure 6. However, the apparent light source does not coincide with the azimuth of the sky sphere as in the indoor setting. As a consequence, the insect would rotate accordingly to ocelli output, its body would be titled and would not be parallel to the ground plane. However, the orientation could be biased to move the apparent light source position to the ground z-axis, by adding an offset to the ocelli output,. This could be possible only if another sensor, such as the compound eyes or simply a gravity sensor, would estimate correctly the ground azimuthal axis. Once the ocelli is biased, it would still respond to sudden changes in attitude due to external disturbances. This attitude estimation strategy is consistent with the observation that the ocelli behave like a high-pass filter, as described above. To this end, we are currently exploring the fabrication of a simple electromechanical gravity sensor that could be used to provide the correct bias for the ocelli, thus allowing the MFI to adapt to a variety of heterogenous and time-varying environments while maintaining high responsiveness to quick external disturbances.

\section{HALTERES}

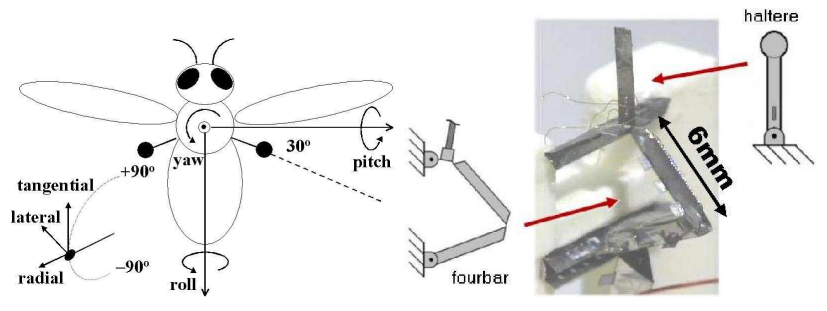

Fig. 7. (a) Schematic of the enlarged halteres; (b) Photo of the completed haltere on a fourbar structure. Adapted from [5]

\section{A. Morphology}

Research on insect flight revealed that in order to maintain stable flight, insects use structures, called halteres, to detect body rotational velocities via gyroscopic forces [19]. The halteres of a fly evolved from hindwings and are hidden in the space between thorax and abdomen so that air current has negligible effect on them (see Figure 7). The halteres resemble small balls at the end of thin rods. There are about 400 sensilla embedded in the flexible exoskeleton at the haltere base. These 
mechanoreceptors function as strain gauges to detect the Coriolis force exerted on the halteres [20]. During flight the halteres beat up and down in vertical planes through an angle of nearly $180^{\circ}$ anti-phase to the wings at the wingbeat frequency. When a fly's halteres are removed or immobilized, it quickly falls to the ground. In addition, the two halteres of a fly are non-coplanar (each is tilted backward from the transverse plane by about $30^{\circ}$ ). This non-coplanarity of the two halteres is essential for a fly to detect rotations about all three turning axes. In fact, a fly with one haltere removed is unable to detect rotations about an axis perpendicular to the stroke plane of the remaining haltere [21].

\section{B. Modeling}

A complex force, as a result of insect motion and haltere kinematics, acts on the halteres during flight [21]. Assuming no translational motion of the insect, this force can be expressed in vector notation by the following:

$$
\mathbf{F}=m \mathbf{g}-m \mathbf{a}-m \dot{\boldsymbol{\omega}} \times \mathbf{r}-m \boldsymbol{\omega} \times(\boldsymbol{\omega} \times \mathbf{r})-2 m \boldsymbol{\omega} \times \mathbf{v}
$$

where $m$ is the mass of the haltere, $\mathbf{r}, \mathbf{v}$, and $\mathbf{a}$ are the position, velocity, and acceleration of the haltere relative to the insect body, $\boldsymbol{\omega}$ and $\dot{\omega}$ are the angular velocity and angular acceleration of the insect, and $\mathbf{g}$ is the gravitational constant. Further, this force can be decomposed into radial, tangential, and lateral components as depicted by the exploded view of the haltere in Figure 7. Insect's body rotations produce centrifugal $(-m \boldsymbol{\omega} \times(\boldsymbol{\omega} \times \mathbf{r}))$ and Coriolis $(-2 m \boldsymbol{\omega} \times \mathbf{v})$ forces on the halteres. The centrifugal force is generally smaller than the Coriolis force and mostly in the radial and tangential directions. Moreover, the centrifugal force is proportional to the square of angular velocity of the insect, it provides no information on the sign of rotations. The Coriolis force, on the other hand, has components in all three directions and contains information on the axis, sign, and magnitude of the insect's body rotations. The angular acceleration force $(-m \dot{\boldsymbol{\omega}} \times \mathbf{r})$ and the Coriolis force are separable because of the $90^{\circ}$ phase shift (they are orthogonal functions). The primary inertial force $(-m \mathbf{a})$ has only radial and tangential components and is orders of magnitude larger than the Coriolis force. The gravitational force $(m \mathbf{g})$ is always constant and depending on the haltere position and the insect's body attitude in space, its distribution in the three directions varies. However, the effect of this gravitational force on the rotation sensing is negligible because it is a tonic lateral component which can be considered as DC offset on the Coriolis force and removed by the subsequent signal processing step.

Figure 8 shows the traces of the components of the Coriolis force for rotations about the roll, pitch, and yaw axes. Note that since the Coriolis force is proportional to the cross product of the angular velocity and the instantaneous haltere velocity, there is no tangential component in the Coriolis force. In addition, to detect body rotations, only the lateral forces on the halteres are measured because the large primary inertial force has no contribution in the lateral direction and hence it is possible to measure the relatively strong Coriolis signal among all other interfering force signals appearing in this direction. Because of the dependence of the Coriolis force on the haltere
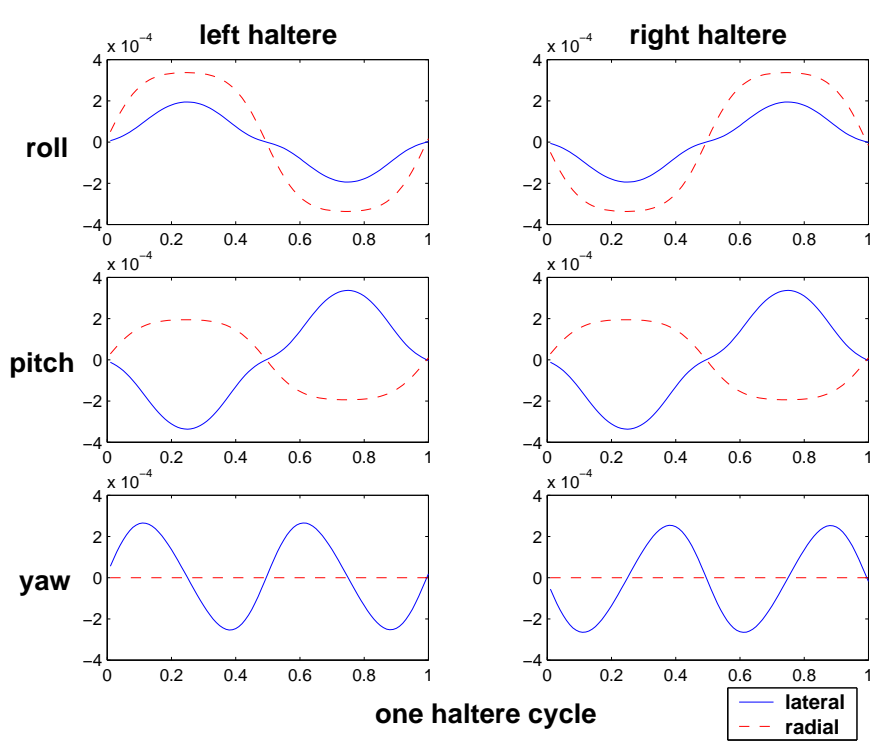

Fig. 8. Coriolis force signals for rotations about the roll, pitch, and yaw axes.

velocity, these force signals are modulated in time with haltere beat frequency. For a roll rotation, the signal is modulated with the haltere beat frequency and the left and right signals are $180^{\circ}$ out-of-phase. For a pitch rotation, the signal is also modulated with the haltere beat frequency, but the left and right signals are in-phase. For a yaw rotation, the signal is modulated with double the haltere beat frequency and the left and right signals are $180^{\circ}$ out-of-phase.

Formally, the Coriolis forces can be obtained by explicitly writing the positions and velocities of the two halteres:

$$
\begin{aligned}
& \mathbf{r}_{l}(t)=\left[\begin{array}{lll}
\sin \alpha \cos \beta(t) & -\cos \alpha \cos \beta(t) & \sin \beta(t)
\end{array}\right]^{T}(18) \\
& \mathbf{r}_{r}(t)=\left[\begin{array}{lll}
\sin \alpha \cos \beta(t) & \cos \alpha \cos \beta(t) & \sin \beta(t)
\end{array}\right]^{T}(19) \\
& F_{l}(t)=-2 m \mathbf{t}_{l}\left[\boldsymbol{\omega} \times \dot{\mathbf{r}}_{l}(t)\right] \\
& F_{r}(t)=-2 m \mathbf{t}_{r}\left[\boldsymbol{\omega} \times \dot{\mathbf{r}}_{r}(t)\right] \\
& \beta(t)=-\Phi \cos 2 \pi \nu t
\end{aligned}
$$

where $\mathbf{r}_{l}(t), \mathbf{r}_{r}(t)$ are the position vectors of the left and right halteres, $F_{l}(t), F_{r}(t)$ are the lateral Coriolis forces measured by the left and right halteres, $\Phi$ is the amplitude of the haltere stroke, $\alpha$ is the tilt angle of the halteres relative to the transverse plane, $\beta(t)$ is the angle between the haltere position $\mathbf{r}$ and the $x-y$ plane, and $\nu$ is the haltere beat frequency. The unit vectors, $\mathbf{t}_{l}=[-\cos \alpha-\sin \alpha 0]$ and $\mathbf{t}_{r}=[-\cos \alpha \sin \alpha 0]$, define the positive (forward) lateral directions of the left and right halteres, respectively (see Figure 7). After some straightforward but tedious manipulation, the measured forces can be written as follows:

$$
\begin{aligned}
F_{l}(t) & =-\left[2 m \sin \alpha f_{1}(t)\right] \omega_{x}+\left[2 m \cos \alpha f_{1}(t)\right] \omega_{y}-\left[2 m f_{2}(t)\right] \omega_{z} \\
F_{r}(t) & =+\left[2 m \sin \alpha f_{1}(t)\right] \omega_{x}+\left[2 m \cos \alpha f_{1}(t)\right] \omega_{y}+\left[2 m f_{2}(t)\right] \omega_{z} \\
f_{1}(t) & =\dot{\beta}(t) \cos \beta(t) ; \quad f_{2}(t)=\dot{\beta}(t) \sin \beta(t)
\end{aligned}
$$

where the modulating signals of the roll $(x)$, pitch $(y)$, and yaw $(z)$ velocities are highlighted in the square brackets and plotted in Figure 8. A careful inspection at these modulating signals reveals specific periodicity and they can be expanded in 


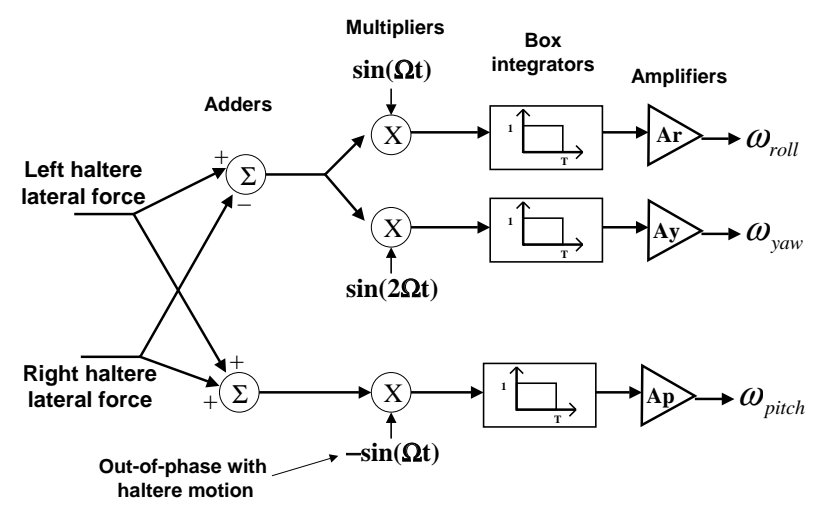

Fig. 9. Demodulation scheme of haltere forces.

Fourier's series as follows:

$$
\begin{aligned}
& f_{1}(t)=\sum_{n=1}^{+\infty} a_{n}(\Phi) \sin ((2 n) 2 \pi \nu t) \\
& f_{2}(t)=\sum_{n=0}^{+\infty} b_{n}(\Phi) \sin ((2 n+1) 2 \pi \nu t)
\end{aligned}
$$

where the coefficients, $a_{n}(\Phi)$ and $b_{n}(\Phi)$, depend on the haltere stroke magnitude $\Phi$. Note that even in the case when the halteres motion is not perfectly sinusoidal, but it is still in phase with the wingbeat frequency, i.e. $\beta(t)=$ $-\sum_{n=1}^{+\infty} c_{n} \cos (2 \pi n \nu)$, the Fourier expansion in Equations (23)-(24) still hold. This includes the case more commonly observed in real insects, where halteres move at constant velocity during upstroke and downstroke, i.e. $\dot{\beta}(t)=$ $v_{\text {max }} \operatorname{sign}(\sin (2 \pi n \nu))$, where $\operatorname{sign}(x)=\frac{x}{|x|}$ and $v_{\max }$ is a constant. This fact is very important, since it highlights one of the robustness properties of the haltere demodulation scheme.

Utilizing the characteristics (frequency, modulation, and phase) of these force signals on the left and right halteres, a demodulation scheme is proposed to decipher roll, pitch, and yaw rotations. First, a pitch rotation can be easily distinguished from roll and yaw rotations by noting the phases of the left and right signals. Because the left and right signals are in-phase for pitch while out-of-phase for roll and yaw, adding the left and right signals retains pitch component and eliminates roll and yaw components. If the left and right signals are subtracted instead, the pitch component is eliminated. The roll angular velocity is distinguished from the yaw angular velocity by noting that the two modulating signals are orthogonal in the Fourier space. In particular, we can extract the first coefficients of the Fourier expansions $a_{1}(\Phi)$ and $b_{0}(\Phi)$ with the following demodulation:

$$
\begin{aligned}
h_{y}(t) & =-\left(F_{l}(t)+F_{r}(t)\right) \sin (2 \pi \nu t) \\
h_{x}(t) & =\left(F_{l}(t)-F_{r}(t)\right) \sin (2 \pi \nu t) \\
h_{z}(t) & =\left(F_{l}(t)-F_{2}(t)\right) \sin (4 \pi \nu t)
\end{aligned}
$$

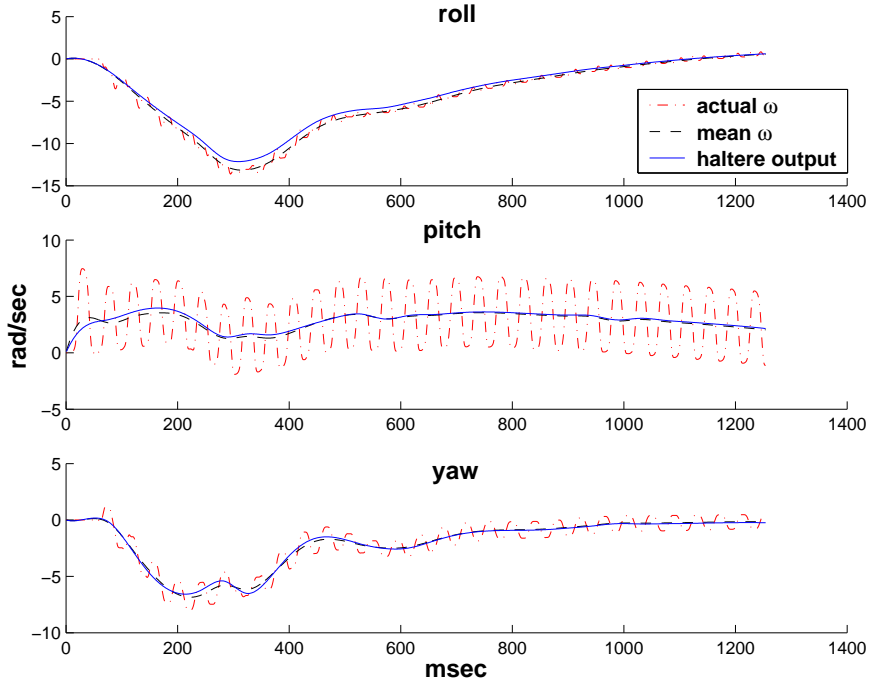

Fig. 10. Angular rotation detection by halteres.

From the above equations, it is clear that when the signals $h_{x}$, $h_{y}$, and $h_{z}$ are averaged over one haltere cycle, a sinusoidal signal at the haltere frequency retrieves the roll component which is modulated with the haltere beat frequency, while a sinusoidal signal at double the haltere frequency retrieves the yaw component which is modulated with double the haltere beat frequency. All higher frequency components are removed by averaging the signals over one haltere cycle:

$$
\begin{aligned}
& y_{1}(t)=\int_{t-\frac{1}{\nu}}^{t} h_{x}(\tau) \mathrm{d} \tau=\frac{2 m a_{1} \sin \alpha}{\nu} \omega_{x}=\frac{1}{A_{x}} \omega_{x} \\
& y_{2}(t)=\int_{t-\frac{1}{\nu}}^{t} h_{y}(\tau) \mathrm{d} \tau=\frac{2 m a_{1} \cos \alpha}{\nu} \omega_{y}=\frac{1}{A_{y}} \omega_{y} \\
& y_{3}(t)=\int_{t-\frac{1}{\nu}}^{t} h_{z}(\tau) \mathrm{d} \tau=\frac{2 m b_{0}}{\nu} \omega_{z}=\frac{1}{A_{z}} \omega_{z}
\end{aligned}
$$

where the constants $A_{x}, A_{y}$, and $A_{z}$ set the gains for the amplifiers. Therefore, this technique effectively decouples roll from yaw. Figure 9 illustrates graphically the proposed demodulation scheme.

\section{Haltere Performance}

The mechanism by which the halteres detect angular velocities and the proposed demodulation method have been tested for the performance of the halteres. The angular velocities of an insect under hovering condition are generated by the Virtual Insect Flight Simulator (VIFS), a software testbed that is used to simulate the dynamics of the MFI and evaluate control algorithms [22], and the results are shown in Figure 10. From the simulation, it is clear that the proposed demodulation scheme, using box integrators as low pass filters, averages out the oscillatory disturbances due to the beating wings. From a flight control's point of view, this is beneficial since the wingbeat kinematics can be controlled at most on a wingbeat-bywingbeat basis. The halteres filter out the periodic oscillations of the angular velocity due to the wing flapping motions. 
TABLE I

COMPARISON OF THE HALTERE TO COMMERCIALLY-AVAILABLE SILICON MICROMACHINED ANGULAR RATE SENSORS.

\begin{tabular}{|c|c|c|c|c|}
\hline & Haltere & $\begin{array}{c}\text { Silicon } \\
\text { MicroRing } \\
\text { Gyro }^{2}\end{array}$ & ADXRS300 & KX210 \\
\hline Weight $(m g)$ & $30^{5}$ & $<600^{6}$ & $<500^{6}$ & $<600^{6}$ \\
Sens. $\left(m V /\left({ }^{\circ} / s\right)\right)$ & 0.1 & 25 & 5 & 6.7 \\
Max Rate $\left({ }^{\circ} / s\right)$ & $\pm 300,000$ & \pm 60 & \pm 300 & \pm 300 \\
B.W. $(H z)$ & 15 & 10 & 40 & 75 \\
Power $(m W)$ & 1 & 75 & 30 & 75 \\
\hline
\end{tabular}

1 Assuming parasitic drive and $1 \%$ duty cycle strain gauge sampling [4].

2 MicroSensors, Inc., http://www.microsensors.com/

3 Analog Devices, Inc., http://www.analog.com/

4 Kionix, Inc., http://www.kionix.com/

5 Including the weight of the fourbar structure.

6 Including the weight of the package.

There are several advantages for the MFI in using halteres instead of MEMS gyroscopes as angular rate sensors. First, the haltere needs very little power since it does not use active actuation. It can be driven parasitically from the wing vibrations when it is placed on the thorax of the MFI. The thorax structure consists of mechanically amplifying fourbar structures actuated by piezoelectric actuators to drive the MFI wings [2] [23][3] (see Figure 7). Second, the haltere has a large dynamic range. It can measure angular velocities from as low as tens of degrees per second to as high as hundreds of thousands of degrees per second, which is often encountered during saccades $\left(90^{\circ}\right.$ turns in less than $100 \mathrm{~ms}$ ) of flying insects. Finally, when the wings are flapping, the MFI body would oscillate, as a result of the wing inertia, along an axis parallel to the wing stroke direction. Since the forces orthogonal to the haltere's beating plane (i.e. lateral forces) are sensed, it is possible to avoid the error caused by this common-mode body oscillation by phaselocking the halteres to the wings in the stroke plane. Table I shows a comparison of the prototype of a mechanical haltere to commercially available MEMS angular rate sensors. Details on halteres design, fabrication and performance can be found in [4].

\section{Attitude Stabilization via Output FeEdBack}

In the previous sections we described how the ocelli can estimate the position of the $z$-axis of the fixed frame relative to the body frame, and how halteres can estimate the insect angular velocities relative to the body frame. In this section we combine the outputs from these two sensory systems to obtain global stabilizing control laws to align the $z$-axis of the body frame with the $z$-axis of the fixed frame. These two axes are aligned if and only if the angle, $\theta$, between them is zero. This angle can be computed from the rotation matrix, $R$, by recalling that the cosine of the angle between two unit vectors is given by their inner product, i.e. $\cos \theta=e_{3}^{T} P_{z}^{a}=\mathbf{e}_{3}^{T} R \mathbf{e}_{3}=r_{33}$ where $P_{z}^{a}$ represents the $z$-axis unit vector of the body frame. Based on the intuition that the input torque should rotate the insect body frame such that the angle $\theta_{z}$ would decrease, we propose the following output feedback law:

$$
\mathbf{u}=-k_{y}\left[y_{2}-y_{1} 0\right]^{T}-k_{\omega} \tilde{\boldsymbol{\omega}}^{b}
$$

where $k_{y}, k_{\omega}$ are scalar and $\tilde{\boldsymbol{\omega}}^{b}$ is the halteres output.
This control law stabilizes the insect orientation as shown in the following two theorems:

Theorem 1. If the light intensity function is $I=f(\theta)=$ $\cos (\theta)$, and $k_{y}, k_{\omega}>0$ then the control law (31) aligns the $z$ axes of the fixed and body frames, i.e. all trajectories of System (1a) approach the set $M=\left\{(R, \boldsymbol{\omega}) \mid P_{z}^{b}=(0,0, \pm 1), \boldsymbol{\omega}=\right.$ $0\}$. However, only the point $M_{1}=\left\{(R, \omega) \mid P_{z}^{b}=\right.$ $(0,0,1), \boldsymbol{\omega}=0\}$ is locally asymptotically stable.

Proof: Substituting control law (31) into Equations (1a), and using Proposition 1, we get:

$$
\begin{aligned}
\dot{R} & =R \hat{\omega} \\
\dot{\boldsymbol{\omega}} & =J^{-1}\left(-k_{y}\left[r_{32}-r_{31} 0\right]^{T}-k_{\omega} \boldsymbol{\omega}+\boldsymbol{\omega} \times J \boldsymbol{\omega}\right)
\end{aligned}
$$

The equilibrium points of this system require that $\boldsymbol{\omega}=0$ and $\mathbf{y}_{O}=0 \Leftrightarrow r_{31}=r_{32}=0 \Leftrightarrow r_{33}=\{-1,1\} \Leftrightarrow P_{z}^{b}=$ $\left(r_{31}, r_{32}, r_{33}\right)=(0,0, \pm 1)$. Let us consider the following Lyapunov function for the set $M_{1}$ :

$V=\frac{1}{2} k_{y}\left[r_{31}^{2}+r_{32}^{2}+\left(1-r_{33}\right)^{2}\right]+\frac{1}{2} \boldsymbol{\omega}^{T} J \boldsymbol{\omega}=k_{y}\left(1-r_{33}\right)+\frac{1}{2} \boldsymbol{\omega}^{T} J \boldsymbol{\omega}$

which is clearly a positive definite function. Its time derivative is given by:

$$
\begin{aligned}
\dot{V}= & -k_{y} \dot{r}_{33}+\boldsymbol{\omega}^{T}[u-\boldsymbol{\omega} \times J \boldsymbol{\omega}] \\
= & k_{y}\left[r_{32}-r_{31} 0\right] \boldsymbol{\omega}-k_{y}\left[r_{32}-r_{31} 0\right] \boldsymbol{\omega}-\ldots \\
& -k_{\omega} \boldsymbol{\omega}^{T} \boldsymbol{\omega}-\boldsymbol{\omega}^{T}(\boldsymbol{\omega} \times J \boldsymbol{\omega}) \\
= & -k_{\boldsymbol{\omega}}\|\boldsymbol{\omega}\|^{2} \leq 0
\end{aligned}
$$

where we used the identity $\mathbf{a}^{T}(\mathbf{a} \times \mathbf{b})=0$. Since the function $V$ satisfies LaSalle's Principle [24], then all trajectories approach the largest invariant set in $S=\{(R, \boldsymbol{\omega}) \mid \dot{V}=0\}$. If we substitute the condition $\boldsymbol{\omega} \equiv 0$ into Equations (32), we find that the largest invariant set in $S$ is $M=\left\{(R, \omega) \mid\left(r_{31}, r_{32}\right)=\right.$ $(0,0), \boldsymbol{\omega}=0\}$, which proves the first part of the theorem. To prove local asymptotical stability for set $M_{1}$, it is sufficient to restrict the set of initial condition to the compact set $\Omega=\left\{(R, \omega) \mid V \leq k_{y}\right\}$. Recalling again LaSalle's Principle, the largest invariant set in $S_{\Omega}=\{(R, \omega) \in \Omega \mid \dot{V}=0\}$ is exactly $M_{1}=\left\{(R, \boldsymbol{\omega}) \mid\left(r_{31}, r_{32}, r_{33}\right)=(0,0,1), \boldsymbol{\omega}=0\right\}$, since $P_{z}^{b}=(0,0,-1) \notin S_{\Omega}$, therefore $M_{1}$ is locally asymptotically stable. The point $M_{2}=\left\{(R, \omega) \mid\left(r_{31}, r_{32}, r_{33}\right)=\right.$ $(0,0,-1), \boldsymbol{\omega}=0\}$ is unstable, but we omit the proof that can be easily obtained by considering the linearized system.

This theorem states that a simple proportional feedback law of the ocelli and halteres outputs can steer the orientation such that the insect $z$-axis will always point toward the light source, i.e. the point $M_{1}$, regardless the initial condition. Although from a theoretical point of view some trajectories converge to the point $M_{2}$, in practice all trajectories converge to the stable point $M_{1}$, since $M_{2}$ is unstable. It is interesting to note that the Lyapunov function is composed of two parts, the kinetic energy $K=\frac{1}{2} \boldsymbol{\omega}^{T} J \boldsymbol{\omega}$ and the potential-like function $U=k_{y} \| P_{z}^{b}-$ $\mathbf{e}_{3} \|^{2}=k_{y}\left(1-r_{33}\right)=1-\cos \left(\theta_{b}\right)$. The potential function is the same as that would arise from a $3 \mathrm{D}$ pendulum in a uniform gravitational field. Therefore, for this particular choice of light intensity function, the ocelli output corresponds to the gradient of a potential function on the sky-sphere. 
The theorem above can be generalized to simply monotonically decreasing light intensity function, although it is necessary to add an additional constraint.

Theorem 2. If the intensity function $I=f(\theta)$ is a differentiable monotonically decreasing function, then there exist positive constants $\lambda>0$ such that for $\left(k_{\omega}>\lambda k_{y}>0\right)$, the control law (31) aligns the z-axes of the fixed and body frames, i.e. all trajectories of System (1a) approach the set $M=\left\{(R, \boldsymbol{\omega}) \mid P_{z}^{b}=(0,0, \pm 1), \boldsymbol{\omega}=0\right\}$. However, only the point $M_{1}=\left\{(R, \boldsymbol{\omega}) \mid P_{z}^{b}=(0,0,1), \boldsymbol{\omega}=0\right\}$ is locally asymptotically stable.

Proof: First, note that Equation (10) can be rewritten as $y_{1}=r_{31} g\left(r_{31}, r_{33}\right), y_{2}=r_{32} g\left(r_{32}, r_{33}\right)$, where $\underline{a} \leq$ $g(x, y) \leq \bar{a}$ according to Equation (15). Define also $\gamma=\frac{\underline{a}+\bar{a}}{2}$ and $\delta=\frac{\bar{a}-\underline{a}}{2}$. The proof of this theorem requires a slightly different choice of Lyapunov function:

$V=\frac{1}{2}\left(\gamma k_{y}+c k_{\omega}\right)\left[r_{31}^{2}+r_{32}^{2}+\left(1-r_{33}\right)^{2}\right]+\frac{1}{2} \boldsymbol{\omega}^{T} J \boldsymbol{\omega}-c\left[r_{32}-y_{31} 0\right] J \boldsymbol{\omega}$

where $c>0$. If the scalar $c$ is small, than the quadratic Lyapunov function is positive definite relative to the point $(\mathbf{z}=$ $\left.P_{z}^{b}-\mathbf{e}_{3}=\left[\begin{array}{lll}r_{31} & r_{31} & 1-r_{33}\end{array}\right]^{T}, \boldsymbol{\omega}\right)$. In fact:

$V \geq \frac{1}{2}\left(\gamma k_{y}+c k_{\omega}\right)\|\mathbf{z}\|^{2}+\frac{1}{2} \sigma_{\min }(J)|| \boldsymbol{\omega}\left\|^{2}-c \sigma_{\max }(J)|| \mathbf{z}\right\||| \boldsymbol{\omega} \mid$

where $\sigma_{\max }(A)$ and $\sigma_{\max }(A)$ are respectively the largest and the smallest eigenvalues for the matrix $A$, and we used the fact that $\left\|\left[r_{32}-y_{31} 0\right]\right\|^{2}=r_{32}^{2}+r_{31}^{2} \leq\|z\|^{2}$. Therefore, the quadratic form is positive definite for $c^{2} \sigma_{\max }^{2}(J)<$ $\sigma_{\min }(J)\left(\gamma k_{y}+c k_{\omega}\right)$, which holds true for $c$ sufficiently small. Let us define the vector $\mathbf{d}=\left[r_{32}-r_{31} 0\right]^{T}$, therefore the time derivative of this Lyapunov function becomes:

$$
\begin{aligned}
\dot{V}= & -\left(\gamma k_{y}+c k_{\omega}\right) \dot{r}_{33}+\boldsymbol{\omega}^{T}[\mathbf{u}-\boldsymbol{\omega} \times J \boldsymbol{\omega}]-\ldots \\
& -c \mathbf{d}^{T}[u-\boldsymbol{\omega} \times J \boldsymbol{\omega}]-c \boldsymbol{\omega}_{b}^{T} J \dot{\mathbf{d}} \\
= & \gamma k_{y} \mathbf{d}^{T} \boldsymbol{\omega}+c k_{\omega} \mathbf{d}^{T} \boldsymbol{\omega}-k_{y}\left[y_{2}-y_{1} 0\right] \boldsymbol{\omega}- \\
& -k_{\omega} \boldsymbol{\omega}^{T} \boldsymbol{\omega}-\boldsymbol{\omega}^{T}(\boldsymbol{\omega} \times J \boldsymbol{\omega})-c k_{y}\left[y_{2}-y_{1} 0\right] \mathbf{d}- \\
& -c k_{\omega} \mathbf{d}^{T} \boldsymbol{\omega}-c \mathbf{d}^{T}(\boldsymbol{\omega} \times J \boldsymbol{\omega})-c \boldsymbol{\omega}^{T} J \dot{\mathbf{d}} \\
= & k_{y}\left(\gamma \mathbf{d}^{T}-\left[y_{2}-y_{1} 0\right]\right) \boldsymbol{\omega}-k_{\boldsymbol{\omega}}\|\boldsymbol{\omega}\|^{2}-c k_{y}\|\mathbf{d}\|^{2}+ \\
& +c \boldsymbol{\omega}^{T} J(\boldsymbol{\omega} \times \mathbf{d})-c \boldsymbol{\omega}^{T} J(\boldsymbol{\omega} \times \mathbf{d}) \\
\leq & -k_{\omega}\|\boldsymbol{\omega}\|^{2}+\delta k_{y}\|\boldsymbol{\omega}\|\|\mathbf{d}\|-c k_{y}\|\mathbf{d}\|^{2}
\end{aligned}
$$

where we used the fact $\boldsymbol{\omega}^{T}(\boldsymbol{\omega} \times J \boldsymbol{\omega})=0$, and that $\left(\mathbf{d}=-\mathbf{e}_{3} \times\right.$ $\left.P_{3}^{b}\right) \Rightarrow \dot{\mathbf{d}}=-\mathbf{e}_{3} \times \dot{P}_{3}^{b}=-\mathbf{e}_{3} \times\left(P_{z}^{b} \times \boldsymbol{\omega}\right)=\left(-\mathbf{e}_{3} \times P_{z}^{b}\right) \times$ $\boldsymbol{\omega}=\mathbf{d} \times \boldsymbol{\omega}=-\boldsymbol{\omega} \times \mathbf{d}$, and that $\left\|\gamma \mathbf{d}^{T}-\left[y_{2}-y_{1} 0\right]\right\|^{2}=$ $r_{31}^{2}\left(\gamma-g\left(r_{31}, r_{33}\right)\right)^{2}+r_{32}^{2}\left(\gamma-g\left(r_{32}, r_{33}\right)\right)^{2} \leq r_{31}^{2} \delta^{2}+r_{32}^{2} \delta^{2}=$ $\delta^{2}\|d\|^{2}$. If $k_{\omega}>\frac{\delta^{2}}{4 c} k_{y}$, then $\dot{V} \leq 0$. Since the Lyapunov function is quadratic and positive definite, it satisfies LaSalle's Principle. The largest invariant set in $S=\{(R, \boldsymbol{\omega}) \mid \dot{V}=0\}=$ $\{(R, \boldsymbol{\omega}) \mid\|\boldsymbol{\omega}\|=0,\|\mathbf{d}\|=0\}$, is $M=S=\left\{(R, \boldsymbol{\omega}) \mid P_{z}^{b}=\right.$ $(0,0, \pm 1), \boldsymbol{\omega}=0\}$. Therefore, all trajectories converge to the set $M$. This proves the first part of the theorem, where $\lambda=\frac{\delta^{2}}{4 c}$. If we restrict the set of initial condition to the compact set $\Omega=$ $\left\{(R, \boldsymbol{\omega}) \mid V \leq \gamma k_{y}\right\}$, we can use La Salle's Principle to claim that the set $\overline{M_{1}}=\left\{(R, \boldsymbol{\omega}) \mid P_{z}^{b}=(0,0,1), \boldsymbol{\omega}=0\right\}$ is locally asymptotically stable, as in the previous theorem.
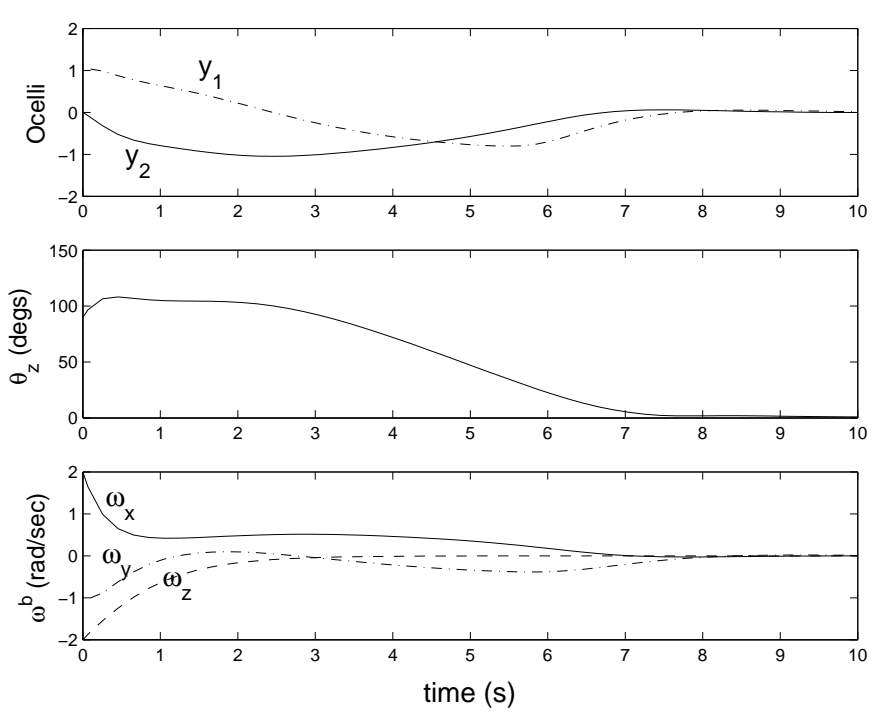

Fig. 11. Simulations results of insect dynamics.

This second theorem states that if the damping gain $k_{\omega}$ is sufficiently large, the dynamics of the insect is slow enough that the field generated by the ocelli feedback $\mathbf{u}_{y}=-k_{y}\left[y_{2}-\right.$ $\left.y_{1} 0\right]^{T}$, steer in practice all the trajectories toward the stable orientation $M_{1}$. However, it is reasonable to ask whether this restrictive condition arises only from the bad choice of potential-like function $U$, which was not obtained considering the light intensity function. More formally, we are interested to know whether there exists a potential-like function $U=U\left(r_{31}, r_{32}, r_{33}\right)$ that gives rise to the torque vector field $\mathbf{u}_{y}=-k_{y}\left[y_{2}-y_{1} 0\right]^{T}$, where $y_{1}$ and $y_{2}$ are defined in Proposition (2). A necessary condition for its existence is that $\nabla U \times P_{z}^{b}=\mathbf{u}_{y}$, where $\nabla U=\left[\frac{\partial U}{\partial r_{31}} \frac{\partial U}{\partial r_{32}} \frac{\partial U}{\partial r_{33}}\right]$. It is easy to verify that this condition implies also $r_{31} y_{2}=r_{32} y_{1}$, which is satisfied only if the light intensity function is $I(\theta)=A \cos (\theta)$, where $A$ is a constant. Therefore, in general a potential-like function that generates the torque vector field $\mathbf{u}_{y}$ does not exist.

It is remarkable that a simple proportional feedback control law based on ocelli and halteres output can reorient the insect toward the light source without knowing the exact light intensity function or the ocelli latitude $h$. Moreover, the set of stabilizing gains $\left(k_{y}, k_{\omega}\right)$ is quite large and they can be optimized relative to some performance metrics, such as settling time or minimal input torque. In particular, the ocelli output can be linearized relative to the stable equilibrium, as shown in Lemma 1. Since the output is linear relative to the variables $\left(r_{31}, r_{32}\right)$, in principle it is possible to design a simple locally stabilizing PD controller based only on ocelli output. This approach is currently under investigation.

\section{Simulations}

Simulations of control law (31) with light intensity function $I=\cos ^{5} \theta$ and initial conditions $\left(\theta_{z}=\frac{\pi}{2}, \boldsymbol{\omega}=[1-22]^{T}\right)$ are shown in Figure 11. As expected, the angle between the $z$-axes as well as the insect angular velocity and the ocelli output go to zero. 
This control law is very promising for three main reasons. First, it is simple: the input control is simply some proportional feedback of the sensor outputs. This is very important in terms of the implementation of control laws, since the MFI has very limited computational power. Second, it is robust: despite its simplicity, this control law does not depend on the exact light intensity function, as long as it is a monotonically decreasing function of the latitude. Third, it is globally stabilizing: remarkably, this control law guarantees the alignment of the insect vertical axis with the light source from any initial condition including the upside down orientation which are likely to occur in the presence of unpredictable wind gusts.

\section{CONCLUSIONS}

In this work we have investigated two types of biologically inspired sensing mechanisms. The halteres and the ocelli have already been fabricated and tested as biomimetic sensors for use on the MFI [4] [5]. We also developed a formal model for ocelli and halteres and proposed a stabilizing attitude control law for the MFI via sensor output feedback. Through our work, we have shown that simple schemes (simple sensor architectures and proportional feedback control) can achieve robust global stability. Finally, we studied the effects on attitude estimation of non ideal light intensity distributions obtained from experiments performed in informative MFI scenarios. We showed that the major consequence was an off-set in the attitude estimation from the ocelli, which could be easily removed with the aid of an additional low-pass filter such as a gravity sensor or the compound eyes.

In the future, we will employ more realistic insect body dynamics that can account for the viscous torques resulting from body rotation, and consider limiting factors such as input torque saturation and control of the torques only on a wingbeat-bywingbeat basis. In addition, we will address the questions on how to design the gains $k_{\omega}$ and $k_{y}$ to improve performance, and how sensor noise affects the performance of the control law. Finally, a simple MFI prototype is under development to test the proposed control schemes.

\section{ACKNOWLEDGEMENT}

The authors wish to thank R.J Wood for designing, building, and testing the ocelli and halteres prototypes. They are also grateful to the valuable comments and criticism of an anonymous reviewer.

\section{REFERENCES}

[1] B. Motazed, D. Vos, and M. Drela. Aerodynamics and flight control design for hovering MAVs. In Proc of American Control Conference, Philadelphia, PA, June 1998.

[2] R.S. Fearing, K.H. Chiang, M.H. Dickinson, D.L. Pick, M. Sitti, and J. Yan. Wing transmission for a micromechanical flying insect. In Proc of the IEEE International Conference on Robotics and Automation, pages 1509-1516, San Francisco, CA, April 2000.

[3] J. Yan, R.J. Wood, S. Avadhanula, R.S. Fearing, and M. Sitti. Towards flapping wing control for a micromechanical flying insect. In Proc of the IEEE International Conference on Robotics and Automation, pages 3901-3908, Seoul, South Korea, May 2001.

[4] W.C. Wu, R.J. Wood, and R.S. Fearing. Halteres for the micromechanical flying insect. In Proc of the IEEE International Conference on Robotics and Automation, Washington, DC, May 2002.
[5] W.C. Wu, L. Schenato, R.J. Wood, and R.S. Fearing. Biomimetic sensor suite for flight control of a micromechanical flight insect: Design and experimental results. In Proc of the IEEE International Conference on Robotics and Automation, Taipei, Taiwan, May 2003.

[6] S. P. Sane and M. H. Dickinson. The control of flight force by a flapping wing: Lift and drag production. Journal of Experimental Biology, 204(204):2607-2626, June 2001.

[7] R.M. Murray, Z. Li, and S.S. Sastry. A Mathematical Introduction to Robotic Manipulation. RCR Press, New York, 1993.

[8] L. Schenato, X. Deng, and S. Sastry. Hovering flight for a micromechanical flying insect: Modeling and robust control synthesis. In 15th IFAC World Congress on Automatic Control, Barcelona, Spain, July 2002.

[9] X. Deng, L. Schenato, and S. Sastry. Identification and attitude control of a micromechanical flying insect. In Seventh International Conference ICARCV, Singapore, Singapore, Dec 2002.

[10] B. Wie. Space vehicle dynamics and control. AIAA Educational Series, Reston, VA, 1998.

[11] Y.T.Y Wen and K. Kreutz-Delgado. The attitude control problem. IEEE Transaction on Automatic Control, 36:1148-62, 1991.

[12] K.D. Bilimoria and B. Wie. Time-optimal three-axis reorientation of a rigid spacecraft. Journal of Guidance, Control, and Dynamics, 16:446452, 1993.

[13] B. Wie and J. Lu. Feedback control logic for spacecraft eigenaxis rotations under slew rate and control constraints. Journal of Guidance, Control, and Dynamics, 18:1372-1379, 1995.

[14] B. Wie, D. Bailey, and C. Heiberg. Rapid multitarget acquisition and pointing control of agile spacecraft. Journal of Guidance, Control, and Dynamics, 25:96-104, 2002.

[15] H. Schuppe and R. Hengstenberg. Optical properties of the ocelli of calliphora erythrocephala and their role in the dorsal light response. Journal of Comparative Biology A, 173:143-149, 1993.

[16] RG Kastberger. The ocelli control the flight course in honeybees. Physiological Entomology, 15:337-346, 1990.

[17] C.P. Taylor. Contribution of compound eyes and ocelli to steering of locusts in flight: I. behavioural analysis. Journal of Experimental Biology, 93:1-18, 1981.

[18] C.P. Taylor. Contribution of compound eyes and ocelli to steering of locusts in flight: Ii. timing changes in flight motor units. Journal of Experimental Biology, 93:19-31, 1981.

[19] R. Hengstenberg. Mechanosensory control of compensatory head roll during flight in the blowfly Calliphora erythrocephala Meig. Journal of Comparative Physiology A, 163:151-165, 1988.

[20] G. Fraenkel and J.W.S. Pringle. Halteres of flies as gyroscopic organs of equilibrium. Nature, 141:919-921, 1938.

[21] G. Nalbach. The halteres of the blowfly Calliphora: I. kinematics and dynamics. Journal of Comparative Physiology A, 173:293-300, 1993.

[22] L. Schenato, X. Deng, W.C. Wu, and S. Sastry. Virtual insect flight simulator (VIFS): a software testbed for insect flight. In Proc of the IEEE International Conference on Robotics and Automation, pages 3885-3892, Seoul, South Korea, May 2001.

[23] M. Sitti, D. Campolo, J. Yan, R.S. Fearing, T. Su, and D. Taylor. Development of PZT and PZN-PT based unimorph actuators for micromechanical flapping structures. In Proc of the IEEE International Conference on Robotics and Automation, pages 3839-3846, Seoul, South Korea, May 2001.

[24] S. Sastry. Nonlinear systems : analysis, stability, and control. Springer, New York, 1999. 